\title{
¿SE PUEDEN ENSEÑAR CONTENIDOS PROCEDIMENTALES EN LAS CLASES DE CIENCIAS?*
}

DE PRO BUENO, A.

Departamento de Didáctica de las Ciencias Experimentales. Universidad de Murcia.

\section{SUMMARY}

The paper we present raises a central question: Can scientific processes be taught in science classes? To be able to answer this initial question some previous issues must first be considered:

Are the learning of processes new in the teaching of Science?

If both researchers and teachers do not conceptualize these ideas in the same way, what then should we understand by the learning of processes?

How are these instructional contents being investigated at present?

Beginning with the identification of research areas in this field, we attempt to answer further questions:

- Are there different levels of complexity in a single process?

- How could we use these ideas in the planning of instructional activities?

To analyse some of these considerations we have used the contents Tabulated Data and Control of Variables.

La reforma del currículo en nuestro contexto educativo ha favorecido que determinadas expresiones pasen a formar parte del acerbo lingüístico de los profesores: ¿Qué pensarían nuestros «maestros del tintero» al escuchar términos como enseñanza constructivista, paradigma $C-T$-S o metacognición? Es cierto que ya se había acuñado el socorrido «cada maestrillo tiene su librillo» como mecanismo de autodefensa; pero, aun así, creemos que tendrían la sensación de estar muy desfasados respecto al momento en que desarrollaron sus labores profesionales. «Menos mal» que se tranquilizarían al ver cómo se enseña en algunas aulas...

Sin duda, la terminología es importante. Los cambios en el lenguaje proyectan modificaciones personales y sociales de ideas, de planteamientos y hasta de formas de pensar, ya que éste es el vehículo más importante de comunicación y de socialización que posee el ser humano. Pero una cosa es cambiar el envoltorio y otra cambiar el regalo... Es preciso conocer no sólo en qué consisten, quién los apoya o qué grado de innovación aportan, sino qué aspectos concretos de mi práctica educativa quiero y puedo cambiar mañana.
Además, esta reflexión no puede limitarse únicamente aI plano personal. Si se quieren introducir modificaciones de mayor significación para el aprendizaje de los alumnos, es importante que los profesores - por lo menos, del mismo centro- sientan una cierta insatisfacción o necesidad por mejorar lo que están haciendo, compartan los significados de estos términos innovadores y tomen decisiones colectivas al respecto.

Con la pretenciosa intención de exponer unas ideas para la discusión y la crítica -pero también para aportar sugerencias que puedan ser útiles a un profesor en su aula- se ha realizado este trabajo.

\section{CONTENIDO PROCEDIMENTAL: ¿UN TÉR- MINO NOVEDOSO?}

Uno de los términos que parece novedoso tras la implantación de la reforma es el de contenido procedimental. En las últimas modificaciones de Ios currículos y no sólo en España, se ha puesto mucho énfasis en este tipo de 
conocimiento académico. Así se puede apreciar en el National Curriculum Council de Inglaterra y Gales, en el proyecto Science for all Americans de Ia AAAS, en los programas de las Junior High School de Alberta, en Canadá, o en las orientaciones de la UNESCO en su Conferencia sobre Educación en Ciencia y Tecnología de París en 1984.

Esto ha motivado que muchos investigadores analicen las consecuencias de estos cambios institucionales: qué exigencias Ileva consigo la incorporación de los contenidos procedimentales al aula (Lucas, 1990), qué piensan los profesores sobre los mismos (Abell y Smith, 1994; Haney et al., 1996; Nott y Wellington, 1996) y, en general, qué elementos innovadores se introducen con la presencia de estos conocimientos respecto a la situación anterior (Buchan y Jenkins, 1992; Roberts, 1995; Galbraith et al., 1997).

Pero no sólo las reformas han incidido en los contenidos procedimentales. También lo han hecho los nuevos enfoques del curículo como ciencia-tecnología-sociedad (Aikenhead y Ryan, 1992; Gilbert, 1992; Roberts, 1995) o ámbitos emblemáticos de la investigación en la enseñanza de las ciencias. Así, podemos ver:

\section{a) Respecto a la utilización de los trabajos prácticos}

La necesidad de revisar el enfoque de este recurso didáctico incide lógicamente en estos conocimientos (Hodson, 1994; Duggan y Gott, 1995; Gott y Duggan, 1996; White, 1996) $\mathrm{y}$, en las revisiones bibliográficas sobre esta temática, se manifiesta inequívocamente la importancia de los procedimientos (Barberá y Valdés, 1996).

También aparecen en investigaciones sobre propuestas metodológicas donde el trabajo práctico juega un papel fundamental (Chang y Lederman, 1994; Watson, 1994; Fraser et al., 1995; Gangoli y Gurumurthy, 1995) o en algunos estudios diagnósticos o exploratorios (Lock, 1992; Doran et al., 1993; Kirschner et al., 1993; Doran et al., 1995) orientados al aprendizaje de estos contenidos.

Incluso, hay abierto un campo muy interesante sobre qué contenidos están implícitos en las actividades prácticas de los libros de texto (Bastida et al., 1990; Tamir y García, 1992; Germann et al., 1996). Esta línea cobra mayor importancia cuando este material pasa a ser el auténtico currículo oficial en la enseñanza obligatoria.

\section{b) Respecto a la resolución de problemas}

Los nuevos enfoques de la resolución de problemas ponen de manifiesto la existencia de una gran variedad de contenidos procedimentales (Siguienza y Saez, 1990; Perales, 1993; Watson, 1994). Quisiéramos resaltar que algunos tradicinalmente parecian ser «patrimonio» del trabajo práctico, lo que llevaba a concepciones y creencias muy discutibles sobre la ciencia y su enseñanza.

También hay otras aportaciones en las que este tipo de conocimientos juega un papel relevante: qué estrategias elaboran los alumnos y cómo las utilizan para dar respuesta a las situaciones problemáticas que se le presentan en el aula (Njoo y Jong, 1993; Langlois et al., 1995; Lee y Fensham, 1996; Lee et al., 1996), qué diferencias caracterizan a los novatos y expertos «resolvedores» de problemas (Zajchowski y Martin, 1993) o qué relación tienen con la capacidad-M en la resolución (Niaz y Robinson, 1992; Niaz, 1996).

\section{c) Respecto al aprendizaje de las ciencias}

Hay contribuciones como resultado de la aplicación de las concepciones sobre de qué forma se produce la adquisición de los contenidos académicos, entre los que obviamente están los procedimentales. Así, además de revisiones generales (Perales, 1993), hay trabajos desde planteamientos muy diversos: el aprendizaje por descubrimiento (Aho et al., 1993; Barron, 1993; Lawson, 1994), Ia psicología evolutiva (Mattheis et al., 1992), el aprendizaje constructivista o como cambio conceptual (Gil, 1993; Furió et al., 1994; Roth, 1994; Martínez y Varela, 1996; Ritchie y Rigano, 1996), el aprendizaje significativo (Colombo et aI., 1991)...

Pero, con tantas aportaciones...

\section{¿QUÉ SON LOS CONTENIDOS PROCEDI- MENTALES?}

No hay una única definición sobre contenidos procedimentales. A continuación se exponen algunas de ellas

«Un procedimiento es un conjunto de acciones ordenadas orientadas a la consecución de una meta[...] En Ios contenidos procedimentales se indican contenidos que también caben bajo la denominación de destrezas, técnicas o estrategias [...]. No debe confundirse un procedimiento con una metodología. El procedimiento es la destreza con la que queremos ayudar a que el alumno construya. Es, por tanto, un contenido escolar objeto de planificación e intervención educativa» (MEC, 1989).

«La Science For All Americans identifica cinco características de los procesos de la ciencia [que deben considerarse en la enseñanza]: la ciencia demanda evidencias; es una combinación de lógica e imaginación; explica y predice; no es autoritaria; y los científicos tratan de identificar y evitar sesgos» (Abell y Smith, 1994).

«El resultado de este proceso de aprendizaje es el conocimiento conceptual-declarativo. Los procedimientos que se utilizan para generar ese conocimiento declarativo se conocen colectivamente como conocimiento procedimental» (Lawson, 1994).

«Denominamos destreza a la aptitud, pericia o habilidad para desempeñar una acción individual específica (comparar, clasificar, observar, informar...). Denominamos estrategia a los procesos mentales complejos, que incluyen destrezas y conceptos, y cuya finalidad es dar solución a una situación problema» (Sevilla, 1994). 
«El conocimiento procedimental se refiere a la habilidad de los alumnos para dar solución a problemas prácticos desde sus propios recursos de destrezas y conceptos sin recetas de un guión o del profesor. Requieren el conocimiento de destrezas y «conceptos de evidencias»[...]. Este término se refiere a todos los conceptos asociados con la evidencia obtenida, incluyendo las relaciones entre las variables y la realidad que representan, la forma de recoger los datos, representarlos e interpretarlos y la noción de probar* lo» (Duggan y Gott, 1995).

«Desde la perspectiva disciplinar, los procedimientos, en calidad de contenidos, conforman el corpus de todas las materias curriculares[...]. Aprender un procedimiento es acceder a una concepción más compleja de la materia[...]. Desde un planteamiento psicopedagógico, podríamos identificar tres funciones básicas: comunicativa y expresiva, cognitiva y metacognitiva, y pragmática. Desde una perspectiva contextual [surge como] la interpretación conjunta [...] entre los alumnos y su profesor, como confrontación entre ambos significados [...].» (Monereo, 1995).

Sin entrar en un contraste detenido entre estas declaraciones, por no constituir un objetivo del trabajo que presentamos, hay que comentar algunas ideas que creemos interesantes.

Un primer aspecto a resaltar es la diferenciación entre un proceso de la ciencia y un contenido procedimental. Como en el caso de los contenidos conceptuales, ni la motivación por aprenderlos, ni el desarrollo madurativo del «usuario», ni la utilidad y finalidad que tiene para el que usa este conocimiento son semejantes. Por lo tanto, habrá que considerar el contexto científico donde tiene su origen, pero será necesario «acondicionarlo» como contenido académico, válido para la formación de cualquier ciudadano y no sólo para físicos, químicos, biớlogos o geólogos.

Otra idea importante que se desprende de las definiciones es que no deben confundirse con actividades de enseñanza. Son contenidos que pueden ser enseñados, como la corriente eléctrica, el mol, la fotosíntesis o la tectónica de placas y, para ello, tendremos que planificar y realizar intervenciones intencionadas que hagan facti. ble su aprendizaje. En este contexto, las actividades deberán actuar, igual que en los conceptuales, como vehículos facilitadores de nuestras intenciones educati. vas; en este caso: aprender a hacer ciencia.

Tampoco se puede olvidar que la separación establecida con los contenidos conceptuales o actitudinales pretende facilitar estudios analíticos o reclamar la atención de los profesores y especialistas. Sin embargo, forman un cuerpo cohesionado de conocimientos que, en conjunto, justifica el valor formativo de estas disciplinas y su inclusión en el currículo. Cuando enseñamos conceptos probablemente también estamos enseñando algunos procedimientos; el problema es cómo lo hacemos, qué aprenden tos alumnos, cómo podríamos enseñar otros...
Por último, quisiéramos hacer notar que la observación o la medición no son los únicos procedimientos ni siquiera los más relevantes de las ciencias o de su enseñanza. Hay un enorme número de habilidades de investigación, de destrezas comunicativas, de estrategias cognitivas... que posiblemente sean generadoras de una mayor cantidad de conocimientos y de aprendizajes. Algunos profesores piensan que los contenidos procedimentales sólo aparecen en las activiđades de laboratorio; creemos que proyectan el desconocimiento del significado de ambos términos.

Como se desprende de todo lo anterior, los contenidos procedimentales ni son nuevos en la enseñanza de las ciencias, ni se aprenden "por casualidad», ni son independientes de los conceptuales, ni se circunscriben a la observación y la medición. Entonces...

\section{¿QUÉ CONTENIDOS PROCEDIMENTALES PÓDEMOS ENSENAR?}

Además de los ya mencionados -observación y medición- hay otros contenidos procedimentales; de hecho, la necesidad de clarificar sus significados y características ha impulsado algunas clasificaciones que nos parecen ilustrativas. En el cuadro I, se han recogido algunas de ellas.

A pesar de los distintos grados de desarrollo, se pueden percibir muchos contenidos semejantes: identificación de problemas, emisión de hipótesis, realización de predicciones, diseño experimental, observación, medición, organización de datos, análisis de datos, comunicación... Hay otros más específicos: confianza en sí mismo, diferenciación cuestión-hipótesis, flexibilidad en los experimentos... Obviamente no son iguales ni pueden serlo, ya que los modelos didácticos, educativos e ideológicos de los autores son diferentes; igual ocurriría si "organizáramos» los contenidos conceptuales de una disciplina.

Quizás sea en las denominaciones más genéricas y en las secuencias internas donde se pongan de manifiesto las diferencias. Así, algunos clasifican los contenidos desde la perspectiva de la resolución de problemas como in. vestigación y comienzan con el reconocimiento de un interrogante o una situación problemática. Otros realizan su clasificación en el contexto de los trabajos prác * ticos aunque con planteamientos antagónicos (hipotético-deductivo o empírico-inductista). Hay quienes los plantean para analizar actividades de enseñanza. También alguno distingue diferentes niveles de dificultad y habla de procesos básicos e integrados.

Como en otros temas de la enseñanza y aprendizaje de las ciencias, hay distintos enfoques y orientaciones. Por lo tanto, no parece lógico ni correcto que se identifiquen los contenidos procedimentales con un único planteamiento metodológico o con una corriente psicopedagógica específica. Desgraciadamente estamos bastante acostumbrados a las etiquetas. 
Cuadro I

Clasificaciones de contenidos procedimentales.

Clasificación de Kirschener, Mester, Middelbeek y Hermans (1993)

Resolución de problemas

- identificar diferentes fenómenos implícitos

- descomponer en problemas pequeños

- emitir hipótesis

- identificar variables y forma de medirlas

- usar datos

\section{Use de destrezas}

- reconocer problemas

- reaccionar frente a resultados imprevistos

- construir modelos

- aplicar principios conocidos

Diseño de experiencias

- diseñar experimentos para el estudio de

fenómenos
- diseñar para contrastar hipótesis

- prever técnicas de medición

- planificar

- conocer limitaciones

- diseñar técnicas de observación

\section{Realización}

- realizar medición

- seguir instrucciones

- usar técnicas de manipulación

- realizar calibrado

- observar cualitativa y cuantitativamente

- ser flexible en los prímeros experimentos

- recoger datos

\section{Interpretación de datos}

- hacer cálculos

- estimar precisión

- aplicar nociones estadísticas
- procesar datos

- interpretar el significado

- evaluar relevancia

- hacer estimaciones

- valorar hipótesis

- evaluar errores de medida

- confirmar hechos y leyes

- analizar datos

- valorar diferencias con los resultados esperados

- incorporar resultados a modelos

Descripción de la experiencia

- hacer sugerencias a partir de resultados

- presentar elementos esenciales

- comunicar hallazgos

- resumir los aspectos

- describir aspectos centrales

- discutir resultados

\section{Clasificación de Lawson (1994)}

\section{Descripción de la naturaleza}

- describir características observables de

jos objetos

- realizar series

- clasificar

- ídern en términos de variables

- identificar variables (continuas y dis-

continuas) y constantes

- medir y realizar gráftcos

- aplicar estadística (media, mediana,

moda)

- diferenciar muestra-población

- predecir probabifísticamente

\section{Planteamiento de cuestiones}

- realizar la cuestión causal a partir de una observación

- diferenciar cuestión-observación-hipótesis interrogativa; cuestiones descriptivas-causales
- reconocer cuestión expositiva

\section{Emisión de hipótesis}

- diferenciar cuestión-hjpótesis: observación-generalización-explicación - reconocer el carácter provisional de las hipotesis

- diferenciar explicación provisional-fenómeno

- generar combinaciones de hipótesis

\section{Predicciones}

- diferenciar predicción-hipótesis

- diseñar experiencias para hipótesis

- seleccionar hipótesis para verificarlas

- diferenciar observación no controladacontrolada

- identificar factor variable independiente y dependiente
- jdentificar factores constantes

- reconocer problemas técnicos de los diseños

- criticar experimentos

Recogida y análisis de datos

- reconocer errores en medidas

- precisión en medida

- organizar datos (tablas, representación gráfica...)

- reconocer elementos comunes en datos; tendencias, extrapolaciones

- relacionar variables

\section{Conclusienes}

- evaluar relevancia de datos y respaldo de hipótesis

- establecer conciusiones

- aplicar conclusiones a situaciones nuevas

\section{Clasificación de Lock (1992)}

Observación

- observación

- lectura de instru-

mentos

\section{Manipulación}

- uso de aparatos

- trabajo sistemático

- realización de ope-

raciones correctas

- seguir instrucciones

\section{Interpretación}

- interpretación,

observación y datos

- fuentes de errores

- habilidad para

hacer cálculos
Planificación

- diseño proceso

experimental

- uso de control

experimental

- resolución de

problemas

\section{Comunicación}

- tenguaje cienti-

fico

- organización de

datos y observa-

ciones

- exactitud de]

proceso

Confianza en si

- búsqueda de aytuda y confirmación
Clasificacion de AAAS (1970)

Procesos básicos Procesos integrados

- Observación

- Relaciones

espacio-tiempo

- Cuantificación

- Medición

- Clasificación

- Comunicación

- Predicción

- Inferencia
- Interpretación de

datos

- Emisión de

hipótesis

- Control de

variables

- Definición

operacional

- Diseño experi-

mental

Clasificación de Tamir y Garcia (1992)

Planificación

- formular preguntas

- predecir resul-

tados

- formular hipo-

tesis

- diseñar proceso

- diseñar expe-

riencia

Realización

- observación

- medición

- manipulación

aparatos

- registro de

resultados

- cálculos numé-

ricos

- trabajo deriva-

do del diseño
Análisis

- tabulación

- representación

grăfica

- inferencias en la

observaciónt

- determinar relaciones

- precistón del experi.

mento

- definir limitaciones

- generalizaciones

- explicación de rela-

ciones

- nuevas preguntas

Aplicación

- predecir sobre la

base de los resultados

- contrastar hipótesis

- aplicar técnicas y

hallazgos 


\section{CONTENIDOS PROCEDIMENTALES}

\section{A. Habilidades de inyestigación}

\section{A.l. Identificación de problemas}

- Conocimiento del motivo del problema.

- Identificación de variables, obtención de datos, contexto...

- Identificación de partes del problema.

- Planteamiento de cuestiones.

\section{A.2. Predicciones e hipótesis}

- Establecimiento de conjeturas contrastables.

- Deducción de prediciones a partir de experiencias, resultados...

- Emisión de hipótesis a partir de un marco teórico.

\section{A.3. Relactones entre variables}

- Identificación de variables (dependiente, independiente...).

- Establecimiento de relaciones de dependencia entre variables.

- Establecimiento de procesos de control y exclusión de variables.

\section{A,4. Diseños experimentales}

- Selección de pruebas adecuadas para contrastar una afirmación.

- Estabjecimiento de una estrategia de resolución de un problema.

A.5. Observación

- Descripción de observaciones y situaciones.

- Representación esquemática de una observación, hecho..

- Identificación de propiedades, características..

- Registro cualitativo de datos.

\section{A.6. Medición}

- Registro cuantitativo de datos.

- Selección de instrumentos de medida adecuados.

- Estimación de medidas sin «medir».

- Estimación de la precisión de un instrumento.

\section{A.7. Clasificación y seriación}

- Utilización de criterios de clasificación.

- Diseño y aplicación de claves de categorización propias.

- Realizacion de series a partir de caracteristicas o propiedades.

A.8. Técnicas de investigación

- Utilización de técnicas elementales para el trabajo de laboratorio.

- Utilización de estrategias básicas para resolución de problemas.

A.9. Trasformación e interpretación de datos

- Organización de datos (cuadros, tablas...).

- Representación de datos (gráficas), extrapolación de datos.

- Interpretación de observaciones, datos, medidas...

\section{A.10. Analisis de datos}

- Formulación de tendencias o relaciones cualitativas.

- Realización de cálculos matemáticos y ejercicios numéricos.

- Identíficación de posibles fuentes de errot.

\section{A.11. Utilización de modelos}

- Uso de modelos analógicos o a escala.

- Uso de fórmulas químicas, de modelos matemáticos y teóricos.

\section{A.12. Elaboracion conclusiones}

- Inferencjas inmediatas a partir de los datos o del proceso.

- Establecimiento de conclusiones, resultados o generalizaciones.

- Juicto crítico de los resultados y del proceso de obtención.

\section{B. Destrezas manuales}

B.1. Manejo de material y realización de monsajes.

- Manipulación del material, respetando normas de seguridad.

- Manipulación correcta de los aparatos de medida.

- Realización de montajes previamente especificados.

B.2.- Construcción de aparatos, maquinas, simulaciones...

\section{Comunicación}

C.1. Anälisis de material escrito o audiovisual

- Identificación y reconocimiento de ideas.

- Inferencia próxima a partir de la información.

- Establecimiento de implicaciones y consecuencias.

\section{C.2. Utilización de diversas fuentes}

- Búsqueda de datos e información en diversas fuentes.

- ldentificación de ideas comunes, diferentes, complementarias...

C.3 Elaboración de materiales

- Informe descriptivo sobre experiencias y procesos vividos.

- Informe estructurado a partir de un guión de preguntas.

- Informe abiezto o ensayo.
No obstante, creemos importante distiguir entre habilidades de investigación, destrezas manipulativas y de comunicación; con ello y los procedimientos asociados, se proyectan creencias y concepciones sobre las ciencias que tienen gran repercusión en su enseñanza. Una clasificación útil para nuestros trabajos aparece en el cuadro II.

Como puede verse, la diversidad de los contenidos, que es una constante en todas las clasificaciones, también lo es en la nuestra. Sin embargo, igual que los conceptos difícilmente se presentan de forma aislada, tampoco parece fácil trabajar procedimientos independientes en un proceso de construcción del conocimiento. Por ello, esta propuesta no pretende excluir la existencia de relaciones lógicas y epistemológicas entre los contenidos diferenciados y, mucho menos, establecer una jerarquía de complejidad o de relevancia didáctica.

Creemos que la utilidad de la realización de este tipo de clasificaciones está en que ayudan a clarificar ideas, o al menos al cuestionamiento de las propias, a reflexionar sobre la práctica educativa... pero, sobre todo, tienen un carácter instrumental. 
Para nosotros, por ejemplo, es una herramienta que facilita el análisis y la reflexión sobre qué contenidos estamos realmente enseñando con nuestras propuestas didácticas o actividades de enseñanza, y cómo podríamos introducir otros o rentabilizar más los que habitual. mente hacemos (Pro, 1995). Así, en el cuadro IIl se ejemplifica cómo un cambio de planteamiento puede mantener los mismos contenidos conceptuales y modificar sustantivamente los procedimentales.

Otra utilidad es la identificación de perfíles de actuación como profesores. En el cuadro IV aparecen cuatro perfi. les reales que pueden servir para trabajar con profesores en formación o en ejercicio -nosotros así lo hemos hecho- y que responden a planteamientos metodológi. cos muy diferentes.

El profesor A realiza las clásicas actividades de un libro de texto en el que aparecen una serie de ejercicios numéricos que ponen el énfasis en la identificación de la fórmula a aplicar y en el reconocimiento de los datos que conocemos en la expresión matemática. Con independencia de otras críticas que pudieran realizarse al respec* to, lo que nos parece preocupante es que, después de trabajar únicamente los contenidos A10 y A12, lección tras lección durante todo el curso, los alumnos no los aprendan...

El profesor B planifica una unidad didáctica después de una "sensibilización encomiable» sobre la importancia de este tipo de contenidos; de esta forma, pasa de considerar dos o tres contenidos en cada actividad a tratar de incluir «todos los posibles». En este caso, hay que recordar que, igual que la inclusion de un gran número de conceptos en una actividad no suele garantizar ni facilitar su aprendizaje, es bastante probable que el alumno «se pierda». Además, las actividades con muchos conte * nidos suelen ser largas, lo que no sólo perjudica «el interés del profesor por acabar los programas» sino que termina aburriendo al alumno. Quizás, pueda ser útil como estrategia de síntesis en el tercio final de una unidad didáctica y, desde luego, no debiera ser tan reiterativa en cuanto a los contenidos implícitos.

El profesor C plantea unas actividades con una distribución posiblemente adecuada en cuanto al número de contenidos implícitos. Sin embargo, proyecta una imagen empírico-inductiva de las ciencias e inconsistente con nuestras concepciones sobre su enseñanza. Como puede verse, los contenidos a enseñar se refieren siempre a observación o međición, manipulación, análisis de datos y establecimiento de conclusiones, lo que pone de manifiesto una visión sesgada del conocimiento científico. Sexía interesante estudiar las auténticas repercusiones para el aprendizaje de esta forma de secuenciar las actividades, pero también para la motivación de los alumnos.

El profesor $D$ realiza una secuencia de actividades en las que aparecen contenidos procedimentales muy variados pero también dispersos. No parece fácil que, por muy brillantes o afortunados que estemos al explicar un concepto, el alumno lo aprenda inmediatamente; normalmente es preciso insistir, cambiar el contexto, relacionarlo y aplicarlo con otros contenidos... Podemos trasladar esta reflexión a los procedimentales

Como puede verse, podemos encontrar perfiles de actua ción muy diferentes que nos permiten reflexionar sobre la práctica educativa. Sin embargo, creemos que no sólo debe orientarse a la investigación o para el diagnóstico de situaciones (toma de conciencia) sino prioritariamen*

\begin{tabular}{|c|c|c|c|}
\hline \multicolumn{2}{|c|}{ Planteamiento 1} & \multicolumn{2}{|c|}{ Planteamiento 2} \\
\hline \multirow{2}{*}{\multicolumn{2}{|c|}{$\begin{array}{l}\text { - Una piedra tiene una masa de } 100 \text { gramos y un volumen de } 50 \\
\text { centímetros cúbicos. ¿Cuál es su densidad? } \\
\text { - Una sustancia tiene una densidad de } 0.5 \mathrm{~g} / \mathrm{cm}^{3} \text {. Si tenemos } 100 \\
\text { gramos de la misma, ¿qué volumen ocupa? }\end{array}$}} & \multirow{2}{*}{\multicolumn{2}{|c|}{$\begin{array}{l}\text { ¿ ¿Cómo podemos medir la masa de un cuerpo? Hazlo con los } \\
\text { cueppos que tienes delante. } \\
\text { - Busca dos cuerpos diferentes que tengan la misma masa. ¿Tienen } \\
\text { el mismo volumen? }\end{array}$}} \\
\hline & & & \\
\hline \multicolumn{2}{|l|}{ - Etcétera. } & \multirow{3}{*}{\multicolumn{2}{|c|}{$\begin{array}{l}\text { - ¿Cómo podemos medir el volumen de este sólido (cubo de made- } \\
\text { ra)? } \\
\text { - Ahora coge dos cuerpos que tengan el mismo volumen. ¿Tienen la } \\
\text { misma masa? } \\
\text { - A la vista de tus experiencias anteriores, ¿se puede decir que los } \\
\text { cuepos que tienen más masa ocupan más volumen? } \\
\text { Etcétera. }\end{array}$}} \\
\hline \multirow{4}{*}{ Contenidos procedimentales } & \multirow{4}{*}{ - Masa, volumen, densidad } & & \\
\hline & & & \\
\hline & & Contenidos conceptuales & - Masa, volumen, densidad \\
\hline & & Contenidos procedimentales & - A.4, A.6, A. 10, A. 12 \\
\hline
\end{tabular}




\begin{tabular}{|l|c|c|c|c|c|}
\hline Prof. A & Act. I & Act. 2 & Act. 3 & Act. 4 & .. \\
\hline Proc. A1 & & & & & \\
\hline Proc. A2 & & & & & \\
\hline Proc. A3 & & & & & \\
\hline Proc. A4 & & & & & \\
\hline Proc. A8 & $\mathrm{X}$ & & & & \\
\hline Proc. A9 & & & & & \\
\hline Proc. A10 & $\mathrm{X}$ & $\mathrm{X}$ & $\mathrm{X}$ & $\mathrm{X}$ & $\mathrm{X}$ \\
\hline Proc. A11 & & & & & \\
\hline Proc. A12 & $\mathrm{X}$ & $\mathrm{X}$ & $\mathrm{X}$ & $\mathrm{X}$ & $\mathrm{X}$ \\
\hline
\end{tabular}

\begin{tabular}{|l|c|c|c|c|c|}
\hline Prof. B & Act. 1 & Act. 2 & Act. 3 & Act. 4 & .. \\
\hline Proc. A1 & $\mathrm{X}$ & $\mathrm{X}$ & $\mathrm{X}$ & $\mathrm{X}$ & $\mathrm{X}$ \\
\hline Proc. A2 & $\mathrm{X}$ & & $\mathrm{X}$ & $\mathrm{X}$ & \\
\hline Proc. A3 & $\mathrm{X}$ & $\mathrm{X}$ & $\mathrm{X}$ & $\mathrm{X}$ & $\mathrm{X}$ \\
\hline Proc. A4 & $\mathrm{X}$ & & $\mathrm{X}$ & $\mathrm{X}$ & \\
\hline Proc. A5 & $\mathrm{X}$ & $\mathrm{X}$ & $\mathrm{X}$ & $\mathrm{X}$ & $\mathrm{X}$ \\
\hline Proc. A6 & $\mathrm{X}$ & $\mathrm{X}$ & $\mathrm{X}$ & $\mathrm{X}$ & $\mathrm{X}$ \\
\hline Proc. A9 & $\mathrm{X}$ & $\mathrm{X}$ & $\mathrm{X}$ & $\mathrm{X}$ & $\mathrm{X}$ \\
\hline Proc. A10 & $\mathrm{X}$ & $\mathrm{X}$ & $\mathrm{X}$ & $\mathrm{X}$ & $\mathrm{X}$ \\
\hline Proc. A12 & $\mathrm{X}$ & $\mathrm{X}$ & $\mathrm{X}$ & $\mathrm{X}$ & $\mathrm{X}$ \\
\hline
\end{tabular}

\begin{tabular}{|l|c|c|c|c|c|}
\hline Prof. C & Act. 1 & Act. 2 & Act. 3 & Act. 4 & $\ldots$ \\
\hline Proc. A1 & & & & & \\
\hline Proc. A2 & & & & & \\
\hline Proc. A3 & & & & & \\
\hline Proc. A5 & $\mathrm{X}$ & & & $\mathrm{X}$ & \\
\hline Proc. A6 & & $\mathrm{X}$ & $\mathrm{X}$ & & $\mathrm{X}$ \\
\hline Proc. A9 & $\mathrm{X}$ & $\mathrm{X}$ & $\mathrm{X}$ & $\mathrm{X}$ & $\mathrm{X}$ \\
\hline Proc. A10 & $\mathrm{X}$ & $\mathrm{X}$ & $\mathrm{X}$ & & $\mathrm{X}$ \\
\hline Proc. A12 & & & $\mathrm{X}$ & $\mathrm{X}$ & \\
\hline Proc. B1 & $\mathrm{X}$ & $\mathrm{X}$ & $\mathrm{X}$ & $\mathrm{X}$ & $\mathrm{X}$ \\
\hline
\end{tabular}

\begin{tabular}{|l|c|c|c|c|c|}
\hline Prof. D & Act. I & Act. 2 & Act. 3 & Act. 4 & .. \\
\hline Proc. A1 & $\mathrm{X}$ & & & & \\
\hline Proc. A2 & & & & & \\
\hline Proc. A3 & & & & & \\
\hline Proc. A4 & & & & & \\
\hline Proc. A6 & & $\mathrm{X}$ & & & \\
\hline Proc. A9 & & & & & \\
\hline Proc. A10 & $\mathrm{X}$ & & $\mathrm{X}$ & & \\
\hline Proc. A12 & $\mathrm{X}$ & & & & \\
\hline Proc. C2 & & & & $\mathrm{X}$ & \\
\hline
\end{tabular}

te para apoyar, si el profesor se encuentra insatisfecho con su enseñanza, otros enfoques metodólogicos que puedan paliar sus problemas.

Por último, hay que señalar que la clasificación mencionada nos ha permitido también identificar y secuenciar los contenidos procedimentales del currículo oficial, analizar y evaluar producciones documentales de los alumnos, delimitar el contenido académico en la planificación de unidades didácticas de los profesores...

Pero, si son tantos contenidos...

\section{¿QUÉ SE ESTÁ INVESTIGANDO SOBRE LOS CONTENIDOS PROCEDIMENTALES?}

Tomando como referencia nuestra clasificación, hemos revisado la producción científica de los últimos años en algunas revistas relevantes para la didáctica de las cien- cias o para nuestro contexto educativo. Se ha tratado de sintetizar esta revisión en los cuadros $\mathrm{Va}, \mathrm{Vb}$ y $\mathrm{Vc}$. La división en habilidades de investigación (integradas y básicas) y destrezas manipulativas y comunicativas se debe solamente a su extensión.

Como puede verse, existe un número importante de trabajos en todos y cada uno de los procedimientos y ámbitos. Lógicamente muchas de las aportaciones no se circunscriben a un sólo contenido, lo que resulta coherente con la relación que hay entre los mismos. No obstante, a pesar de las singularidades propias de cada uno, es posible distinguir líneas y objetivos comunes de investigación:

- necesidad de clarificar el significado de algunos contenidos procedimentales;

- reconocimiento del papel y aportación de estos contenidos al denominado concepto de evidencia o a la resolución de problemas; 
Cuadro Va

Ejemplos de investigaciones en habilidades de investigación integradas.

\begin{tabular}{|c|c|}
\hline CONTENIDO & TEMATICAS INVESTIGADAS \\
\hline $\begin{array}{l}\text { Identificación } \\
\text { de problemas }\end{array}$ & $\begin{array}{l}\text { - Presentación de problemas y modelo de definición de problemas (Sigüenza y Sáez, 1990; Álvarez, 1996) } \\
\text { - Nuevos problemas a partir de la resolución de problemas (Aho et al., l993; Varelas,1996) } \\
\text { - Identificación de variables y parámetros de un problema en una simulación computerizada (Njoo y Jong, 1993) } \\
\text { - Comunicación entre alumnos para identificar y diseñar estrategias de resolución (Roth, 1994) } \\
\text { - Planteamiento y estrategia de resolución de problemas prácticos (Watson, 1994) } \\
\text { - Influencia de la formulación de problemas en una actividuad intelectual (Langlois et al., 1995) } \\
\text { - Identificación, estrategias de resolución y partes de un problema (Lee y Fensham, 1996) } \\
\text { - Variables cognitivas que influyen en la identificación de problemas (Lee et al., 1996) }\end{array}$ \\
\hline $\begin{array}{l}\text { Predicciones } \\
\text { e hipótesis }\end{array}$ & 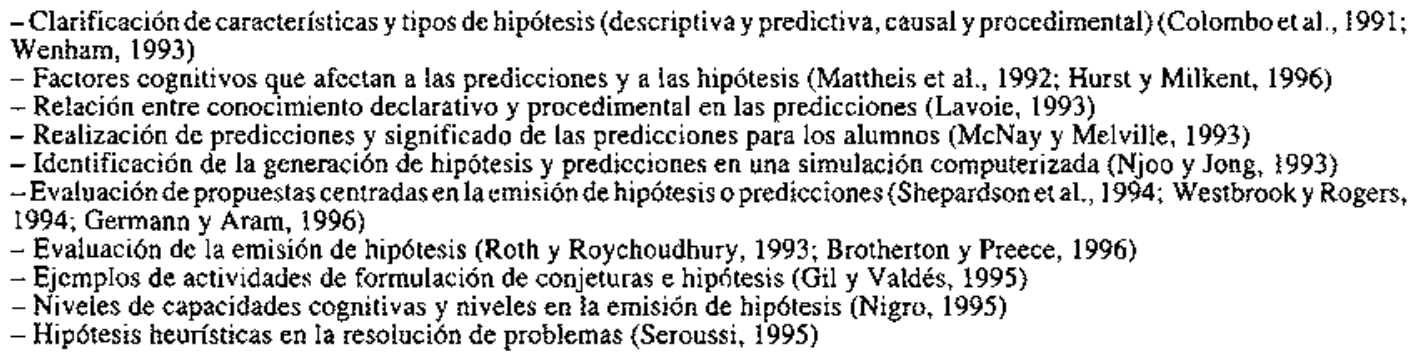 \\
\hline $\begin{array}{l}\text { Relación entre } \\
\text { variables }\end{array}$ & $\begin{array}{l}\text { - Inferencias de causalidad y de no-causalidad en alumnos (Acevedo et al., 1990) } \\
\text { - Establecimiento de jcrarquías en la relación entre variables (Daniels y Braund, 1992) } \\
\text { - Factores cognitivos que afectan a la identificación o relación de variables (Mattheis et al., 1992; Stavy y Tirosh, 1996) } \\
\text { - Requerimientos para el control de variables (Song y Black, I992) } \\
\text { - Identificación de distintos niveles en la relación entre variables en el sonido (Linder, 1993) } \\
\text { - Evaluación de la relación entre variables (Roth y Roychoudhury, 1993; Westbrook y Rogers, 1994; Brotherton y Preece, } \\
\text { 1996) } \\
\text { - Dificultades en las interacciones entre variables o en su identificación (Zohar, 1995; Duggan et al., 1996) } \\
\text { - Conceptos de evidencia y relación entre variables en el trabajo práctico (Gott y Duggan, 1996; Park y Pak, 1997) }\end{array}$ \\
\hline $\begin{array}{l}\text { Diseños } \\
\text { experimentales }\end{array}$ & $\begin{array}{l}\text { - Establecimiento de jerarquías en el diseño de experiencias (Daniels y Braund, 1992) } \\
\text { - Factores cogntitivos que afectan al diseño de experiencias (Mattheis et al., 1992) } \\
\text { - Evaluación del diseño de experiencias (Doran et al., 1993; Roth y Roychoudhury, 1993; Doran et al., 1995) } \\
\text { - Identificación del diseño de experimentos en una simulación computerizada (Njoo y Jong, 1993) } \\
\text { - Comunicación entre alumnos para diseñar experiencias (Roth, 1994) } \\
\text { - Ejemplos de actividades de diseño de investigaciones (Gil y Valdés, 1995) } \\
\text { - Identificación de los patrones y exigencias del diseño de experiencias (Germann et al., 1996a) }\end{array}$ \\
\hline
\end{tabular}

- identificación de dificultades y establecimiento de niveles y exigencias jerárquicas en cada uno;

- justificación de la inclusión de los contenidos procedimentales en Ia enseñanza obligatoria;

- estudio de las influencias de factores cognitivos, cognoscitivos, sociales... en su aprendizaje;

- diseño y aplicación de instrumentos que permitan evaluar el aprendizaje de los mismos; en relación con la evaluación de las habilidades integradas, aparece de forma relevante el uso del ordenador;

- búsqueda de situaciones de clase que favorecen la adquisición de estos conocimientos;

- en menor medida, estudio de la relación con los contenidos conceptuales y actitudinales.
Desde nuestro punto de vista, creemos que la profundización en estas líneas de trabajo puede favorecer no sólo la entrada de elementos innovadores en el aula sino un cambio profundo en la propia investigación en la didáctica de las ciencias. No obstante, como no podemos olvidar las pretensiones y limitaciones de este trabajo, nos vamos a centrar en uno de los interrogantes señalados.

\section{¿EXISTEN NIVELES DE COMPLEJIDAD EN UN CONTENIDO PROCEDIMENTAL?}

Muchas veces, cuando se analizan actividades de enseñanza o se explicita qué se va a impartir en una unidad diđáctica, sólo se indica si están presentes una serie de contenidos procedimentales. Parece como si la presencia o la ausencia fueran los únicos elementos a conside. rar de cara a la enseñanza o a su aprendizaje. 
Cuadro $\mathrm{Vb}$

Ejemplos de investigaciones en habilidades de investigación básicas.

\begin{tabular}{|c|c|}
\hline CONTENIDO & TEMÁTICAS INVESTIGADAS \\
\hline Observación & $\begin{array}{l}\text { - Evaluación de la observación (Al Busaidi y Lock, 1992; Doran et al., 1993,1995; Brotherton y Preece, 1996) } \\
\text { - Evaluación de propuestas con papel importante de la observación (Brickhouse, 1994; Shepardson et al., 1994) } \\
\text { - La observación en la secuencia de resolución de problemas prácticos (Watson, 1994) } \\
\text { - Papel de la observación en el curriculo (Duggan y Gott, 1995) } \\
\text { - Exigencias de la observación (Lucas, 1995) } \\
\text { - Descripción y representación de fa observación (Díaz y Jiménez, 1996) } \\
\text { - Concepto de evidencias y papel de la observación (Park y Pak, 1997) }\end{array}$ \\
\hline Medición & $\begin{array}{l}\text { - Evaluación de la medición (Al Busaidi y Lock, 1992; Doran et al., 1993, Doran et al., 1995; Gangoli y Gurumurthy, 1995; } \\
\text { Brotherton y Preece, 1996) } \\
\text { - Comunicación entre alumnos para recoger datos (Roth, 1994) } \\
\text { - La medicion en la secuencia de resolución de problemas prácticos (Watson, 1994) } \\
\text { - Papel de la medicion en el curriculo (Duggan y Gott, 1995) } \\
\text { - Dificultades en la recogida de datos (Duggan et al., 1996) } \\
\text { - Conceptos de evidencia y papel de la medición (Germann y Aram, 1996; Gott y Duggan, 1996; Varelas, 1996) } \\
\text { - Ideas de los alumnos sobre la fiabilidad de tos datos experimentales (Lubben y Millar, 1996) }\end{array}$ \\
\hline $\begin{array}{l}\text { Organización } \\
\text { e } \\
\text { interpretación } \\
\text { de datos }\end{array}$ & 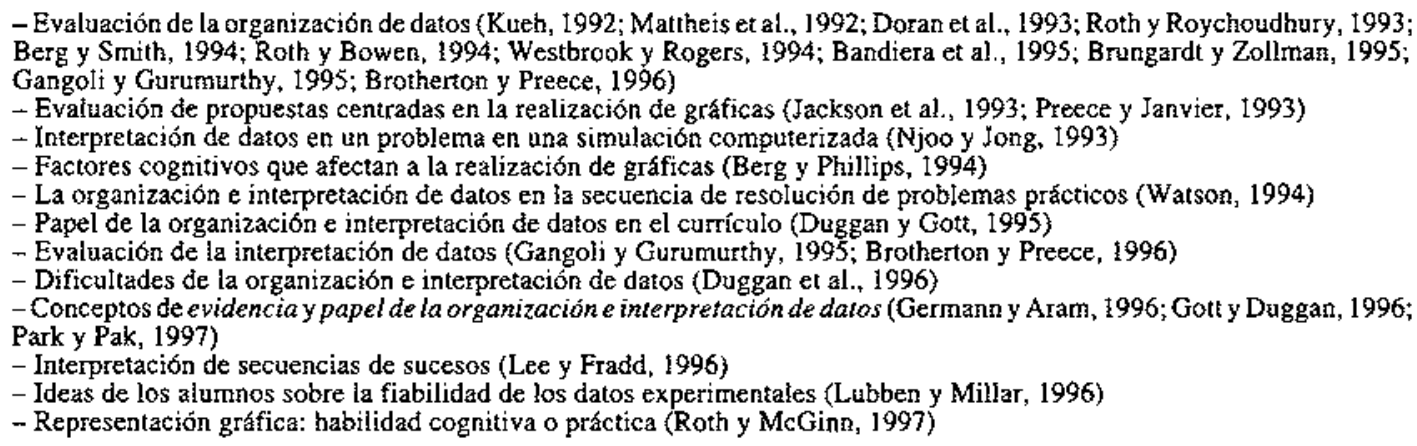 \\
\hline $\begin{array}{l}\text { Analisis } \\
\text { de datos }\end{array}$ & $\begin{array}{l}\text { - Evaluación del análisis de datos (Kueh, 1992; Doran et al., 1993; Jackson et al., 1993; Njoo y Jong, 1993; Preece y Janvier, } \\
\text { 1993; Roth y Roychoudhury, 1993; Roth y Bowen, 1994; Brotherton y Preece, 1996) } \\
\text { - Factores cognitivos que afectan al análisis de datos (Mattheis et al., 1992; Stavy y Tirosh, 1996) } \\
\text { - Descripción de estrategias de resolución (Zajchowski y Martin, 1993; Serousi, 1995; Niaz, 1996) } \\
\text { - Comunicación entre alumnos para analizar datos (Roth, 1994) } \\
\text { - El análisis de datos en la secuencia de resolucion de problemas prácticos (Watson, 1994) } \\
\text { - Conceptos de evidencia y papel del análisis de datos (Germann y Aram, 1996; Gott y Duggan, 1996) }\end{array}$ \\
\hline $\begin{array}{l}\text { Utilización } \\
\text { de modelos }\end{array}$ & $\begin{array}{l}\text { - Utilización y construcción de modelos en la resolución de problemas (Borrego et al., 1993; Abell y Roth, 1995; Hafner y } \\
\text { Stewart, 1995; Hamison y Treagust, 1996; Penner et al., 1997) } \\
\text { - Uso de analogías por los profesores de ciencias (Harrison y Treagust, 1993; Dagher, 1995; Treagust et al., 1996) }\end{array}$ \\
\hline $\begin{array}{l}\text { Elaboracion } \\
\text { de } \\
\text { conclusiones }\end{array}$ & $\begin{array}{l}\text { - El establecimiento de conclusiones en la resolución de problemas (Aho et al, 1993; Watson, 1994) } \\
\text { - Evaluación de conclusiones (Roth y Roychoudhury, 1993; Doran et al., 1995; Brotherton y Preece, 1996) } \\
\text { - Establecimiento de conclusiones en un problema en una simulación computerizada (Njoo y Jong, 1993) } \\
\text { - Conceptos de evidencia y papel de las conclusiones (Germann y Aram, 1996) } \\
\text { - Conclusión en una estrategia de resolución (Niaz, 1996) }\end{array}$ \\
\hline
\end{tabular}

Sin embargo, nuestra práctica diaria nos dice que no es lo mismo medir con una regla que hacerlo con un amperímetro, observar los objetos de una clase que utilizar un telescopio, identificar las variables en un problema "semicerrado" que en una situación problemática abierta... Estas diferencias de dificultad deben considerarse en la secuenciación de contenidos en el currículo (Pro, 1997) o en las taxonomías sobre la interacción de los alumnos con el medio o en la identificación de los esquemas de comprensión de las ciencias (Shayer y Adey, 1984).

Parece necesario buscar alguna estrategia que permita establecer estos niveles de complejidad con el fin de 
Cuadro Vc

Ejemplos de investigaciones en destrezas manipulativas y comunicativas.

\begin{tabular}{|c|c|}
\hline CONTENIDO & TEMÁTICAS INVESTIGADAS \\
\hline $\begin{array}{l}\text { Manejo de } \\
\text { material y } \\
\text { realización } \\
\text { de montajes }\end{array}$ & $\begin{array}{l}\text { - Evaluación de la manipulación de aparatos y montajes (Al Busaidi y Lock, I992; Doran et al., 1993, Doran et al., 1995; } \\
\text { Gangoli y Gurumurthy, 1995) } \\
\text { - Utilización de medios audiovisuales en la resolución de problemas (Leonard, 1992; Jackson et al., 1993; Njoo y Jong, I993; } \\
\text { Simmons y Lunetta, 1993; Brungardt y Zollman, 1995) } \\
\text { - La manipulación en la secuencia de resolución de problemas prácticos (Watson, 1994) } \\
\text { - Papel de la manipulación en el currículo (Duggan y Gott, 1995) } \\
\text { - Dificuitades de la manipulación (Duggan et al., 1996) } \\
\text { - Conceptos de evidencia y papel de la manipulación de aparatos (Got y Duggan, 1996) } \\
\text { - Ideas y niveles de los alumnos sobre la fiabilidad de datos medidos (Lubben y Millar, 1996) }\end{array}$ \\
\hline $\begin{array}{l}\text { Análisis } \\
\text { y utilización } \\
\text { de material }\end{array}$ & $\begin{array}{l}\text { - Características de los ejercicios incluidos en textos, manuales... (Bastida et al., 1990; Tamir y García, 1992; Germann et } \\
\text { al., 1996b) } \\
\text { - Destreza de razonamiento con la utilización de material escrito (Strang y Shayer, 1993; Keys, 1994: 1995; Koch y Eckstein, } \\
\text { 1995; Nigro, 1995) } \\
\text { - Dificultades lingǘsticas (Holiday et al., 1994; Lee et al., 1995; Lee y Fradd, 1996; Grayson, 1997) }\end{array}$ \\
\hline Comunicación & $\begin{array}{l}\text { - Técnica V de Gowin para la comunicación en el trabajo experimental (Novak, 1991; Izquierdo, 1994; Del Carmen, } 1995 \text {; } \\
\text { Calvet, 1997) } \\
\text { - Comunicación verbal según diferentes dinámicas de clase (Pizzini y Shepardson, 1992; Gayford, 1993) } \\
\text { - Factores cognitivos en la comunicación (Strang y Shayer, 1993) } \\
\text { - Elaboración de material escrito (Keys, 1994, 1995; Muñoz, 1995; Sanmarti, 1997) } \\
\text { - La comunicación en la secuencia de resolución de problemas prácticos (Watson, 1994) } \\
\text { - Patrones en los diseños escritos de experiencias (Germann et al., 1996a) } \\
\text { - Construcción del conocimiento a partir de la comunicación (Aho et al., 1993; Varelas, 1996) }\end{array}$ \\
\hline
\end{tabular}

tomar decisiones como to hacemos respecto a cualquier contenido objeto de enseñanza (criterios de selección, secuenciación en los programas, inclusión en las actividades, evaluación...).

Aunque no hemos encontrado aportaciones específicas en la literatura científica sobre esta problemática, creemos que hay tres factores a considerar: en qué consiste el contenido procedimental, en qué contexto va a ser utilizado y qué prerrequisitos tiene su aprendizaje.

Ya hemos ejemplificado esta idea con la observación y la medición (Pro, 1997), pero vamos a hacerlo en este trabajo con dos contenidos procedimentales distintos; uno de ellos se incluye en las habilidades de investigación básicas (tabulación de datos) y el otro en las integradas (control de variables).

\section{A. Niveles de complejidad en la tabulación}

Para establecer los niveles correspondientes al contenido procedimental de la tabulación, hay que distinguir previamente entre tablas de frecuencias y tabla de correspondencias de datos entre distintas variables. Aunque lógicamente tienen elementos comunes, no se realizan las mismas operaciones cognitivas si tenemos que relacionar una variable con la frecuencia de cada uno de sus valores (por ejemplo, tabla de frecuencia de las diferentes plantas que hay en una parcela) que si debe- mos establecer correspondencias entre datos de diferentes variables (por ejemplo, tabla de espacios y tiempos en el estudio del movimiento de un objeto).

En cualquier caso, siguiendo nuestro modelo, creemos que hay que reflexionar sobre las operaciones cognitivas que los caracterizan, el contexto de los datos que van a tabularse y los prerrequisitos.

\section{A.1. Tabulación de frecuencias}

Los elementos comunes a un proceso cualquiera de tabulación de frecuencias podrían ser:

- ser consciente que una variable puede tomar diferentes valores y percibir la utilidad de organizarlos (utilidad);

- identificar los diferentes valores de la variable (identificación);

- interpretar el significado científico que tienen valores iguales y diferentes en una variable (interpretación);

- si son comparables los datos (escalas ordinal y cuantitativa), ordenarlos según su valor; si son nominales establecer un orden para su ubicación en la tabla (ordenación);

- introducir el concepto de frecuencia y encontrar la frecuencia de cada dato (reiteración); 


\section{Cuadro VI}

Niveles de complejidad de la tabulación de frecuencias.

\begin{tabular}{|c|c|}
\hline Niveles & Elementos a considerar en la enseñanza de la tabulación de frecuencias \\
\hline \multirow[t]{3}{*}{1} & Referencia: Los datos son fácilmente identificables y son de escala nominal \\
\hline & $\begin{array}{l}\text { - Reconocer que una variable puede tomar diferentes valores } \\
\text { - Percibir la utilidad de organizarlos por la frecuencia de los datos } \\
\text { - Identificar los diferentes valores de la variable } \\
\text { - Interpretar el significado científico del mismo valor en la variable y de valores diferentes }\end{array}$ \\
\hline & $\begin{array}{l}\text { 1.2 Introducir el concepto de frecuencia; encontrar la frecuencia de cada dato } \\
\text { - Reconocer las «partes» de la tabia: ubicación de datos y frecuencia } \\
\text { - Realizar la tabla de frecuencias }\end{array}$ \\
\hline \multirow[t]{3}{*}{2} & Referencia: Los datos son fácilmente identificables, son de escala ordinal o cuantitativa y no hay agrupamientos \\
\hline & - Repetir secuencia 1.1 \\
\hline & $\begin{array}{l}2.2 \quad \text { Ordenar los datos según su valor } \\
\text { - Encontrar la frecuencia de cada dato } \\
\text { - Realizar la tabla de frecuencias }\end{array}$ \\
\hline \multirow[t]{3}{*}{3} & Referencia: Los datos son fácilmente identificables, son de escala ordinal o cuantitativa y hay agrupamientos \\
\hline & - Repetir secuencia 2.1 y 2.2 del nivel 2 \\
\hline & $\begin{array}{l}3.2 \text { - Percibir la utilidad de agrupar datos; fijar los criterios de agrupamiento } \\
\text { - Encontrar las frecuencias de categorias o intervalos } \\
\text { - Realizar la tabla de frecuencias }\end{array}$ \\
\hline \multirow[t]{3}{*}{4} & Referencia: Los datos no son fäcilmente identiftcables, son de cualquier escala y no hay agrupamientos \\
\hline & $\begin{array}{l}\text { 4.1 - Revisar y buscar, si ha lugar, los datos problemáticos } \\
\text { - Tomar decisiones en cuanto a los datos de partida }\end{array}$ \\
\hline & 4.2 - Repetir la secuencia de los niveles I (si es de escala nominal) o 2 (si es ordinal o cuantitativa) \\
\hline 5 & Etcétera. \\
\hline
\end{tabular}

- si es necesario, fijar los criterios de agrupamiento y encontrar las frecuencias de categorías o intervalos (agrupación);

- realizar la tabia de frecuencias (ejecución).

Por otro lado, Ios elementos del contexto donde puede ser utilizada la tabulación de frecuencias podrían ser: el tipo de escala utilizada en la recogida de los datos, Ia facilidad para reconocerlos (con independencia de la forma de obtenerlos) y la necesidad de agruparlos o no en categorías o intervalos.

Por último, los prerrequisitos necesarios para este tipo de tabulación serf́an: conocer el concepto de variable (que puede tomar diferentes valores), ser capaz de identificar algunas conceptual o experiencialmente, y ser consciente de la existencia y características de diferentes escalas (por lo menos, las que se vayan a utilizar).
Si combinamos estos tres aspectos, se pueden establecer los diferentes niveles de complejidad que genéricamente tendría este contenido procedimental; así se ha realizado el cuadro VI.

Como veremos más adelante los elementos de cada nivel son referentes que podemos utilizar en la elaboración de actividades de enseñanza, pero además creemos que aportan criterios para la secuenciación de este contenido procedimental en el currículo.

Así, por ejemplo, parece aconsejable que, para enseñar la tabulación de frecuencia (que, por muy fácil que nos parezca a los profesores, el alumno no nace sabiendo este contenido) inicialmente se realice con valores teóricos fácilmente identificables y no con datos recogidos experimentalmente donde existe un margen de «inseguridad" que puede condicionar otros aspectos. Tampoco interesa, al principio, trabajar con un número grande de 
Cuadro VH

Niveles de complejidad de la tabulación de datos de varias variables.

\begin{tabular}{|c|c|c|}
\hline Niveles & & Elementos a considerar en la enseñanza de la tabulación de datos \\
\hline \multirow[t]{5}{*}{1} & \multicolumn{2}{|r|}{ Referencia: Datos fácilmente identificables; dos variables: una de escalo nominal y otra cuantitativa } \\
\hline & 1.1 & $\begin{array}{l}\text { - Identificar que las dos variables pueden tomar diferentes valores } \\
\text { - Percibir la utilidad de organizarios por cotrespondencia entre datos de distintas variables } \\
\text { - Identificar los diferentes valores de las variables; interpretar el significado científ too de los valores }\end{array}$ \\
\hline & 1.2 & $\begin{array}{l}\text { - Identificar la variable nominal } \\
\text { - Introducir el concepto de correspondencia; encontrar el par de cada dato } \\
\text { - Reconocer las «partesn de la tabla: ubicación de datos de la nominal y de sus pares en la cuantitativa } \\
\text { - Realizar la tabla de datos }\end{array}$ \\
\hline & 1.3 & $\begin{array}{l}\text { - Identificar la variable cuantitativa; ordenar sus vatores de menor a mayor } \\
\text { - Reconocer las «partes» de la tabia: ubicar los datos ordenados de la cuantitativa y buscar sus pares } \\
\text { - Realizar la tabla de datos }\end{array}$ \\
\hline & 1.4 & $\begin{array}{l}\text { - Comparar las dos tablas e interpretar que representan las mismas correspondencias } \\
\text { - Revisar el proceso seguido }\end{array}$ \\
\hline \multirow[t]{4}{*}{2} & \multicolumn{2}{|r|}{ Referencia: Datos facilmente identificables; dos voriables de escala cuantitativa } \\
\hline & 2.1 & - Repetir secnencia 1.1 del nivel 1 \\
\hline & 2.2 & $\begin{array}{l}\text { - Elegir la primera variable (¿independiente?) y ordenar los datos obtenidos en la misma } \\
\text { - Encontrar los valores que corresponden a cada dato de la primera variable } \\
\text { - Reconocer las «partes» de la tabla; realizar la tabla de datos }\end{array}$ \\
\hline & 2.3 & - Elegir la segunda variable como ¿independiente? y repetir la secuencia 1.3 y 1.4 del nivel 1 \\
\hline \multirow[t]{2}{*}{3} & \multicolumn{2}{|r|}{ Referencia: Datos fácilmente identificables; tres variables de escala cuantitativa } \\
\hline & 3.1 & - Repetir secuencia del nivel 2 y adaptarla al caso de tres variables \\
\hline \multirow[t]{4}{*}{4} & \multicolumn{2}{|r|}{ Referencia: Dos variables de escala cuantitativa; para un valor de una hay varios de otra (por ejemplo, tres) } \\
\hline & 4.1 & - Repetir secuencia 1.1 del nivel 1 \\
\hline & 4.2 & $\begin{array}{l}\text { - Elegir la variable con un solo valor ( } \text { independiente?); ordenar sus datos según su valor } \\
\text { - Encontrar los valores que corresponden a cada dato de la primera variable en la otra; si para un dato de la primera } \\
\text { variable hay varios diferentes de fa otra, tomar decisiones estadisticas } \\
\text { - Identificar los «nuevos y únicos valores» de la segunda variable y realizar la tabla de datos }\end{array}$ \\
\hline & 4.3 & - Repetir 1.4 con especial revisión de las decisiones estadísticas \\
\hline 5 & \multicolumn{2}{|c|}{ Etcétera. } \\
\hline
\end{tabular}

valores que requiera procesos largos y tediosos para conocer cada frecuencia y, mucho menos, que aconseje la utilización de intervalos o categorías para simplificar la organización de los datos. No parecen excesivamente complejos los prerrequisitos, por lo que se deberían abordar varios niveles en educación primaria (seguro que lo agradecerán los profesores de secundaria).

$\mathrm{Y}$, desde luego, el alumno debe ver alguna utilidad -hecho que suele darse por supuesto- a este contenido y reconocer las diferentes operaciones cognitivas (identificación, interpretación, reiteración...) que lleva consigo, antes de «automatizarlas».

\section{A.2. Tabulación de datos de diferentes variables}

Este contenido procedimental es muy habitual en las ciencias pero no es exclusivo, por lo que es posible que se haya irabajado en otras disciplinas del currículo. En 
cualquier caso, pueden existir diferencias en las variables, en la forma de medirlas, en sus características... por lo que posiblemente sea recomendable revisar los conocimientos existentes y retomarlos en nuestro contexto.

Con independencia de esta circunstancia, seguiremos un proceso análogo al anterior. Lo primero sería establecer los elementos comunes a procesos de tabulación de datos correspondientes a distintas variables; estos podrían ser:

- ser consciente que distintas variables pueden tomar diferentes valores y percibir la utilidad de organizarlos (utilidad);

- identificar los diferentes valores de las variables e interpretar su significado científico (identificación $y$ diferenciación);

- elegir la primera variable o la variable independiente;

- si son comparables los datos (escalas ordinal y cuantitativa), ordenar los que corresponden a esta primera variable de forma creciente; si son nominales, establecer un orden para su ubicación en la tabla (ordenación);

- introducir el concepto de correspondencia y encontrar los datos de las demás variables que se corresponden con los de la primera (correspondencia);

- si para un dato de la primera variable hay varios de otra, tomar decisiones estadísticas; si hay pares incompletos, hacerlo notar (decisión);

- realizar la tabla de datos (ejecución).

Por otro lado, los aspectos que contextualizan la tabulación de datos podrían ser: el tipo de escala de las variables, la facilidad para ei reconocimiento de los datos (incluyendo si se trata o no de correspondencias biyectivas) y el número de variables. Aunque es posible encontrar otros, no queremos profundizar más en el proceso para facilitar su explicación.

Por último, los prerrequisitos necesarios para este tipo de tabulación serían muy similares a los del contenido procedimental anterior: conocer el concepto de variable (que puede tomar diferentes valores), ser capaz de identificar algunas conceptual o experiencialmente y conocer la existencia y características de escalas (por lo menos, las que se vayan a utilizar).

De una combinación de las operaciones cognitivas características, del contexto de aplicación y de la consideración de los prerrequisitos, se obtienen los niveles que aparecen en el cuadro VII.

Alguien podría pensar -no exento de razón-que, si hay que utilizar esta secuencia cada vez que se haga una tabla de datos, se ralentizaría mucho el «desarrollo de los programas». Al respecto, quisiéramos señalar que tanto los niveles como los procesos asociados constituyen contenidos de toda la educación obligatoria. Una enseñanza adecuada de las ciencias implicaría que los alumnos, al comienzo de la ESO, deberían tener superados como mínimo los tres primeros niveles. Y si ya lo sabe el alumno, ¿para qué vamos a enseñárselos de nuevo?

Ahora bien, si la realidad no es ésta, el problema no está ni en las exigencias cognitivas de los conocimientos ni en las capacidades de los estudiantes ni en el tiempo que precisa su enseñanza. Otros profesores habrán optado por no enseñarlos en los niveles anteriores del sistema educativo (aunque esté contemplado en los programas oficiales).

También se pueden inferir consecuencias para la secuen. ciación de contenidos: al principio trabajar con escalas cuantitativas prioritariamente (excepto al introducir el nivel 1) o comenzar con correspondencias biyectivas,... $y$, por supuesto, no suponer que el alumno le va a ver utilidad o va a ser consciente de la secuenciación de las operaciones cognitivas.

\section{B. Niveles de complejidad del control de variables}

Desde nuestra perspectiva, uno de los contenidos procedimentales más interesantes y útiles que puede ser ense * ñado en la educación secundaria es, sin duda, el control de variables. Posiblemente, sin acceder al significado de este conocimiento, es difícil entender el origen de las numerosas relaciones causales, estructuras conceptuales, leyes fenomenológicas, estrategias experimentales... que tienen las ciencias, que han posibilitado su desarrollo histórico y que justifican su enseñanza. Pero, como cualquier otro conocimiento, no se suele adquirir «por casualidad» y es preciso identificar pasos en su aprendizaje.

Siguiendo la mecánica utilizada en la tabulación, para establecer los niveles correspondientes habría que con* siderar el propio proceso de control de variables, los contextos en los que puede realizarse y los prerrequisitos, que veremos que tienen una gran importancia.

Los elementos comunes a cualquier control de variables podrían ser:

- identificar la variable dependiente de un problema; identificar posibles variables independientes (identificación);

- reconocer y desechar las variables independientes que sean combinación lineal de otras variables;

- encontrar estrategias o técnicas que permitan variar o fijar las variables dependiente e independientes (manipulación de variables);

- fijar un plan de actuación: establecer las combinaciones posibles de variables independientes (estrategia de actuación);

- elegir una combinación, fijar variables controles; variar la variable independiente y recoger variaciones de la dependiente (relación);

- estudiar si existe regularidad en la relación (regularidad); 
Cuadro VIII

Niveles de complejidad del control de variabjes.

\begin{tabular}{|c|c|c|}
\hline Niveles & & Elementos a considerar en la enseñanza del control de variables \\
\hline \multirow[t]{4}{*}{ I } & \multicolumn{2}{|r|}{$\begin{array}{l}\text { Referencia: Problema con tres variables ( } 1 \text { variable dependiente y } 2 \text { variables independientes), con escalas ordinales o } \\
\text { nominales, variación y fijación fáciles de las variables independientes y con relaciones directas }\end{array}$} \\
\hline & 1.1 & $\begin{array}{l}\text { - Identificar la variable dependiente de un problema } \\
\text { - Identificar las dos variables independientes de dicho problema; reconocer que no son combinación lineal } \\
\text { - Encontrar estrategias que permitan apreciar la variación de la variable dependiente } \\
\text { - Encontrar estrategias para variar o fijar las variables independientes } \\
\text { - Fijar un plan de actuación: establecer las dos combinaciones posibles de las variables independientes }\end{array}$ \\
\hline & 1.2 & $\begin{array}{l}\text { - Elegir primera combinación, fijar la variable control; variar la variable independiente y ver las variaciones de la variable } \\
\text { dependiente } \\
\text { - Analizar los resultados de la relación en esta primera combinacion } \\
\text { - Estudiar si existe regularidad en esta primera relación } \\
\text { - Establecer conclusiones cualitativas a partir de los resultados (relación directa) de esta primera combinacion } \\
\text { - Elegir la otra combinación, fijar la variable control; variar la variable independiente y ver las variaciones de la variable } \\
\text { dependiente en este caso } \\
\text { - Analizar los resultados de la relación en esta segunda combinación } \\
\text { - Estudiar si existe regularidad en la relación de esta segunda combinación } \\
\text { - Establecer conclusiones cualitativas a partir de los resultados (relación directa) }\end{array}$ \\
\hline & 1.3 & $\begin{array}{l}\text { - Establecer conclusiones globales a partir de las parciales de cada combinación } \\
\text { - Revisar el proceso seguido }\end{array}$ \\
\hline \multirow[t]{4}{*}{2} & \multicolumn{2}{|r|}{$\begin{array}{l}\text { Referencia: Problema con tres yariables ( } 1 \text { variable dependiente y } 2 \text { variables independientes), con escalas cuantitativas, } \\
\text { variación y fijación fáciles y con una relación directa y otra sin relación. }\end{array}$} \\
\hline & 2.1 & - Repetir el mismo proceso que en 1.1 \\
\hline & 2.2 & $\begin{array}{l}\text { - Elegir una de las combinaciones, fijar la variable control; variar la variable independiente y ver las vartaciones de la } \\
\text { variable dependiente } \\
\text { - Suponiendo que no exista relación, estudiar si existe regularidad } \\
\text { - Establecer conclusiones cualitativas a partir de los resultados de esta combinación } \\
\text { - Repetir el mismo proceso que en } 1.2 \text { en la relación directa }\end{array}$ \\
\hline & & - Repetir el mismo proceso 1.3 \\
\hline 3 & \multirow{2}{*}{\multicolumn{2}{|c|}{$\begin{array}{l}\text { Referencia: Problema con tres variables (l variable dependiente y } 2 \text { variables independientes), con escalas nominales y } \\
\text { cuantitativas, variacion fácil y con una relación inversa, como minimo } \\
\text { - Repetir } 1.1 \text {, adaptar } 1.262 .2 \text { y repetir } 1.3\end{array}$}} \\
\hline & & \\
\hline 4 & \multicolumn{2}{|r|}{$\begin{array}{l}\text { Referencia: Problema con cuatro variables ( } I \text { variable dependiente y } 3 \text { variables independientes), con escalas cuantitativas, } \\
\text { variación fácil y con existencia o no de relación }\end{array}$} \\
\hline 5 & \multicolumn{2}{|c|}{ Etcétera } \\
\hline
\end{tabular}

- analizar los resultados de la variable dependiente respecto a la independiente estudiada (análisis);

- establecer conclusiones a partir de los resultados (conclusión);

- establecer conclusiones globales a partir de las parciales de cada combinación (relación entre conclusiones);

- revisar el proceso seguido (revisión).

Por otro lado, el contexto donde puede ser aplicado es más diverso y complejo que en otros procedimientos pero, al menos, habrá que considerar: el tipo de escala de las variables, el número de variables independientes, la existencia o no de relación, si la relación es directa o inversa y si la calidad de los datos de referencia facilita el estudio de este contenido.

Los prerrequisitos, como en cualquier habilidad de investigación integrada, son más complejos. Además de haber superado algunos niveles de relación entre variables (de los que no nos ocuparemos en este trabajo), el alumno debe ser capaz de:

- reconocer las posibles variables (y sus escalas) que están implícitas en un problema y ser consciente de la importancia de establecer relaciones entre ellas; 
- a partir de una serie de variables, realizar todas las posibles combinaciones de relaciones entre ellas, tomándolas de dos en dos;

- reconocer las variables independientes (v.i.) y la varia* ble dependiente (v.d.) en cada una de las relaciones estabiecidas anteriormente;

- ser capaz de discutir cualitativamente la posibilidad o no de relaciones en cada una de las combinaciones, utilizando en la argumentación hechos de la vida cotidiana;

- encontrar las variables que son combinación lineal de otras y otras que no lo son.

Una combinación de estos tres aspectos nos permite establecer unos niveles para el contenido procedimental del control de variables como los que aparecen en el cuadro VIII.

Como puede intuirse, si son consistentes estos niveles y sus correspondientes operaciones cognitivas, el proceso de enseñanza y aprendizaje de este tipo de contenidos es largo y no se puede circunscribir a un curso o a una lección. Esto nos ratifica en la poca relevancia que tiene hablar de contenidos procedimentales en términos de presencia o ausencia.

Por otro lado, las exigencias de los prerrequisitos (sobre todo, que el alumno sea capaz de identificar variables independientes que no sean combinaciones lineales) hacen aconsejable que se introduzcan en el currícuio más tarde, por ejemplo, que los de tabulación; por lo tanto, los premequisitos pueden utilizarse en muchos casos como criterios de secuenciación «inter-contenidos».

No obstante, al terminar la educación primaria, deberíamos haber conseguido que fueran capaces de establecer relaciones causales que no se basen únicamente en ob. servaciones, reconocer relaciones lineales entre dos variables, distinguir los aspectos distintos de una misma realidad... Probablemente no sepan controlar variables pero están creadas las condiciones para su aprendizaje a lo largo de la educación secundaria.

También podemos apreciar: la importancia de que el alumno sea consciente de las correspondientes operacio" nes cognitivas (identificación, manipulación de variables, relación, regularidad...); la conveniencia de que las primeras relaciones sean cualitativas para evitar que se convierta en un proceso de inferencia matemática; la necesidad de una directividad del profesor, pues no es un contenido que se pueda aprender autonomamente; la revisión del proceso seguido que permita al estudiante reconocer los pasos dados y valorar la estrategia utilizada...

Lógicamente esto no conlleva un método concreto de enseñanza o unas actividades únicas pero sí unas coorde. nadas de actuación que se apoyen en que son contenidos formativos para los alumnos, tienen sus reglas de aprendizaje, $y$, con independencia de las exigencias adminis- trativas, estamos convencidos que debemos enseñarlos (aunque, como dicen algunos profesores, sólo sea para mejorar los conocimientos conceptuales).

Pero, además de ayudar en las decisiones para la secuenciación de contenidos en el currículo.

\section{¿Cómo SE PUEDen UTILIZAR ESTAS IDEAS EN EL DISENO DE ACTIVIDADES?}

Para nosotros, las actividades son las unidades de organización de la estrategia de enseñanza que concretan en la práctica educativa las creencias, teorías, principios... que tenemos los profesores. Su importancia es incuestionable, pues integran decisiones del tipo: qué contenidos vamos a enseñar, cómo contemplaremos y usaremos los conocimientos que ya tiene el alumno, qué situaciones utilizaremos para favorecer el aprendizaje...

Es cierto que esta «atomización» del proceso de enser̃anza olvida otra faceta muy importante: la relación. entre las actividades en una unidad didáctica o una lección. Pero dejaremos para otros trabajos estas apreciaciones más holísticas y nos centraremos en la utilidad de las ideas anteriores para el establecimiento de una secuencia de enseñanza - primer paso de cara al diseño de actividades.

En efecto, pensamos que, detrás de una actividad, hay unos contenidos conceptuales, procedimentales y actitudinales que aparecen relacionados. El aprendizaje de éstos o su utilización en nuevos ámbitos del conocimiento (que es otro nivel de aprendizaje...) requiere explícita o implícitamente una serie de operaciones cognitivas que llevan asociadas los contenidos de forma independiente y otros mecanismos de interrelación que permite un aprendizaje conjunto de los mismos. Por lo tanto, el punto de partida en el diseño de una actividad debe ser considerar las características asociadas a los contenidos -en nuestro caso, la secuencia de enseñanza del procedimiento-, aunque lógicamente aquí no acaba.

Desde este planteamiento, el proceso a seguir pasaría por:

a) la identificación del contenido académico concreto que tratamos de enseñar y de la secuencia genérica asociada;

b) la concreción del contexto de aplicación; con ello, quedaría determinado un nivel de complejidad;

c) el establecimiento de la secuencia de enseñanza correspondiente a dicho nivel.

Vamos a ejemplificarlo con dos contenidos procedimentales diferentes, dejando a un lado las relaciones conceptuales que indudablemente se ponen en juego; por ejemplo, realizar una tabla de datos sobre variables que afectan a la evaporación de agua a la atmósfera. 


\section{Cuadro IXa}

Posible secuencia para enseñar una zabla de datos.

Tabla de datos sobre variables que afectan la evaporación de agua a la atmósfera

- Identificar las variables que intervienen (por ejemplo: núm. de experiencia, temperatura ambiente, superficie de exposición, volumen de agua existente, masa evaporada).

- Percibir la utilidad de organizarlos en una tabla de datos.

- Identificar los diferentes valores de las variables; interpretar el significado de valores que se repiten en una variable o que son diferentes (mayores o menores).

- Elegir la variable independiente: núm. de experiencia; ordenarlos como experiencia 1, experiencia 2, experiencia 3...

- Encontrar los valores que corresponden a cada dato de la primera variable en las demás (T ambiente, superficie, volumen y masa de la experiencia 1 ; igual en la experiencia $2 ; \ldots$.).

- Reconocer las «partes» de la tabla: ubicación de datos de la escaia nominal (experiencia) en la primera columna y los pares correspondientes en las demás.

- Realizar la tabla de datos.

Cuadro IXb

Posible secuencia para un control de variable.

Factores que afectan a la velocidad de caída de un cuerpo

- Identiffcar la velocidad de caída como variable dependiente del problema.

- Identificar las variables independientes de dicho problema (por ejemplo, la altura y la masa).

- Reconocer que estas variables independientes no son combinación lineal entre sí.

- Encontrar estrategias que permitan apreciar la variación de la velocidad (por ejemplo, a partir del tiempo que está en el aire).

- Encontrar estrategias para variar o fijar la altura y la masa del objeto.

- Fijar plan de actuación: a) un cuerpo (misma masa) se deja caer desde distintas alturas.

b) dos cuerpos de distinta masa se dejan caer desde la misma altura.

- Elegir opción $a$ : fijar la v. control (misma masa), variar la v.j. (altura) y ver las variaciones de lá v.d. (tiempo que está en el aire).

- Analizar los resultados de esta primera combinación.

- Estudiar si existe regularidad en esta primera relación con un cuerpo de distinta masa, lanzándolo a distintas aituras.

- Establecer conclusiones cualitativas y cuantitativas a partir de los resultados (relación directa) de esta primera combinación.

- Elegir opción $b$ : fijar la v. control (misma altura), variat la v.i. (dos cuerpos de distinta masa) y ver las variaciones de la v.d. (tiempo que está en el aire).

- Analizar los resultados de la rełación en esta primera combinación (no hay relación).

- Estudiar si existe regularidad lanzando los cuerpos a otra altura.

- Establecer conclusiones cualitativas a partir de los resultados (no hay relación).

- Establecer conclusiones globales (puede llegarse a la conclusión de que existe una relación directa entre altura y velocidad, $\mathrm{v}^{2}=2 \mathrm{~g} \mathrm{~h}, \mathrm{y}$ no existe con la masa).

- Revisar el proceso. 
Suponemos que ya disponemos de los datos de temperatura ambiente, superficie de exposición, volumen de agua existente en el recipiente y la masa evaporada, correspondientes a varias experiencias. En este caso suponemos que son facilitados al alumno y que éste no tiene problemas en identificarlos (por ejemplo, si los debe obtener experimentalmente, buscando en diversas fuentes...).

Todo esto corresponde al contenido procedimental que hemos denominado tabulación de datos de distintas variables. Con ello, tendremos una secuencia genérica de operaciones cognitivas que lleva implícito este conocimiento y a las que anteriormente aludimos: utilidad, identificación y diferenciación, ordenación, correspondencia y ejecución.

También resulta fundamental considerar el contexto de aplicación, de ahí nuestro siguiente paso. Se trata de cinco variables (una nominal y cuatro cuantitativas) y donde, para cada dato de la escala nominal, las demás variables sólo tienen un valor; por lo tanto, podría considerarse en el nivel 3 de la tabulación de datos.

Considerando los elementos a tener en cuenta para la enseñanza en este nivel del cuadro VII, hemos plasmado en el cuadro IX los diferentes pasos que podrían conternplarse en el diseño de una actividad que lleve implícito este contenido.

Si estamos hablando de una situación de iniciación al aprendizaje de un contenido novedoso, habrá que poner el énfasis en cada uno de los pasos que aparecen en la secuencia; no podemos olvidar que el alumno no aprende estos conocimientos autónomamente. Ahora bien, cuando empieza a interiorizarse, el profesor puede obviar algunos pasos; no porque cambie la secuencia sino porque el alumno o el usuario comienza a automatizar su utilización.

Veamos otro ejemplo: estudiar qué factores afectan a la velocidad de caída de un cuerpo. Si planteamos esta cuestión a nuestros alumnos, suelen aparecer implícita o explícitamente la masa y la altura; normalmente, si están acostumbrados a una dinámica participativa en la cons. trucción de sus conocimientos, también aparecen otras variables pero sólo utilizaremos éstas para facilitar Ia ejemplificación.

Siguiendo el proceso anterior, corresponde al contenido procedimental que hemos denominado control de yariables $\mathrm{y}$, con ello, tendremos una secuencia genérica de operaciones cognitivas (identificación, manipulación de variables, estrategia, relación, regularidad, análisis, conclusiones, revisión del proceso).

El segundo paso es considerar el contex to de aplicación. Se trata de variables cuantitativas fácilmente medibles: la dependiente puede ser el tiempo de caída y las independientes serían la altura y la masa (no son combinación lineal entre sí). Dos son las posibles combinacio nes: fijar la altura y dejar caer dos objetos de distinta masa desde esa altura, y lanzar un mismo cuerpo desde diferentes alturas. Como precaución añadida todos los objetos deben tener una forma similar; con estas carac* terísticas, podría considerarse en el nivel 2.

En base a ello, se ha desarrollado la secuencia de ense. ñanza de este contenido procedimental que aparece en el Cuadro X, que obviamente habrá que plasmar en formu. laciones y planteamientos específicos dependiendo de los modelos y creencias de los profesores o de las características de los alumnos.

Como hemos dicho, cuando se ha utilizado alguna vez, el proceso es más «ágil» y no es preciso enfatizar tanto en la sistemática de cada secuencia. Esto no es nuevo: cuando unos contenidos conceptuales, como la ley de Ohm o la conservación de la masa en una reacción, son estudiados por primera vez, requieren una serie de pasos que faciliten sil incorporación a la estructura cognitiva de los alumnos. Sin embargo, una vez asumidos como conocimientos, no hace falta demostrarlos con cada circuito o reacción química que nos encontremos. Lo que sí nos puede interesar es su utilización en nuevas situaciones para ampliar su significado; análogamente podríamos decir de los contenidos procedimentales.

\section{¿SE PUEDEN ENSEÑAR CONTENIDOS PRO. CEDIMENTALES EN LAS CLASES DE CIEN- CIAS?}

Éste era el título de nuestro trabajo y la pregunta que ha planeado en el mismo. Nuestra respuesta es clara: Por supuesto que sí, pero resulta más complejo de lo que algunos dan a entender.

\section{AGRADECIMIENTO}

Las ideas y materiales que aparecen en este trabajo han sido utlizados en los cursos de formación desarrollados en el proyecto de investigación PS 94-0177 subvencionado por la DGICYT.

\section{NoTA}

* Ponencia presentada en el V Congreso Internacional sobre Investigación en la Didáctica de las Ciencias (Murcia, 10 a 13 de septiembre de 1997). 


\section{REFERENCIAS BIBLIOGRÁFICAS}

AAAS (1970). Science- a process approach commentary for teachers. AAAS: Xerox Corporation.

ABELL, S. y ROTH, M. (1995). Reflections on a Fifth-Grade life science lesson: making sense of children's understanding of scientific modeIs. Int. Jour. Science Education, 17 (1), pp. 59-74.

ABELL, S. y SMITH, D. (1994). What is science?: preservice elementary teachers' conceptions of the nature of science. Int. Jour. Science Education, 16 (4), pp. 475-487.

ACEVEDO, J.A. (1990), Razonamiento causal en una tarea de contexto natural. Un estudio evolutivo con estudiantes de bachillerato. Investigación en la Escuela, 10, pp. 61-70.

AHO, L., HUOPIO, J. y HUTTUNEN, S. (1993). Learning science by practical work in Finnish Primary Schools using materials familiar from the environment: a pilot study, lnt. Jour. Science Education, 15 (5), pp. 497-507.

AIKENHEAD, G. y RYAN, A. (1992). The development of a new instrument: "Views on Science-Technology-Society" (VOSTS). Science Education, $76(5)$, pp. 477-491.

AL BUSAIDI, R. y LOCK, R. (1992). Assessment of science practical skills in Omani 12th-grade students. Int. Jour. Science Education, 14 (3), pp. 319-330.

ÁLVAREZ, R. (1996). Las controversias científicas. Sus implicaciones didácticas y su utilidad mediante un ejemplo: la controversia sobre la edad de la Tierra. Alambique, 8, pp. 63-69.

BANDIERA, M., DUPRE, F., IANNIELLO, M. y VICENTINI, M. (1995). Una investigación sobre habilidades para el aprendizaje científico. Enseñanza de las Ciencias, 13(1), pp. $46-54$.

BARBERÁ, O. y VALDÉS, P. (1996). El trabajo práctico en la enseñanza de las ciencias: una revisión. Enseñanza de las Ciencias, 14 (3), pp. 365-379.

BARRÓN, A. (1993). Aprendizaje por descubrimiento: principios y aplicaciones inadecuadas. Enseñanza de las Ciencias, 11(1), pp. 3-11.

BASTIDA, M.F., RAMOS, F. y SOTO, J. (1990). Prácticas de laboratorio: ¿una inversión poco rentable? Investigación en la Escuela, 11, pp. 77-91.

BERG, C. y PHILLIPS, D. (1994). An investigation of relationship between logical thinking structures and ability to construct and interpret line graphs. Jour. Res. Science Teaching, 31 (4) pp. 323-344

BERG, C. y SMITH, P. (1994). Assessing students' abilities to construct and interpret line graphs: disparities between multiplechoice and free-response instruments. Science Education, 78 (6), pp. $527-554$.

BORREGO, M.J. et al. (1993). EI aprendizaje de los métodos de estudio indirectos aplicados al contenido de la estructura de la Tierra en el bachillerato. Investigación en la Escuela, 20, pp. 103-110.

BRICKHOUSE, N. (1994). Children's observations, ideas and development of classroom theories about light. Jour. Res. Science Teaching, 31 (6), pp. 639-656.

BROTHERTON, P. y PREECE, P. (1996). Teaching science process skills. Int. Jour. Science Education, 18 (1), pp. 65-74.
BRUNGARDT, I. y ZOLLMAN, D. (1995). Influence of interactive videodisc instruction using simultaneous-time analysis on kinematics graphing skills of High School Physics students. Jour. Res. Science Teaching, 32 (8), pp. 855- 869.

BUNCHAN, A. y IENKINS, E. (1992). The internal assessment of practical skills in science in England and Wales, 19601991: some issues in historical perspective. Int. Jour. Science Education, 14 (4), pp. 367-380.

CALVET, M. (1997). La comunicación escrita en el trabajo experimental. Alambique, 12, pp. 63-73.

COLOMBO, L.,SALINAS, J. y PESA, M. (1991). La generación autonoma de «conflictos cognoscitivos» para favorecer cambios de paradigmas en el aprendizaje de la física. Enseñanza de las Ciencias, 9 (3), pp. 237-242.

CHANG, H. y LEDERMAN, N. (1994). The effect of levels of cooperation within physical science laboratory groups on physical science achievement. Jour. Res. Science Teaching, 31 (2), pp. $167-181$

DAGHER, Z. (1995). Analysis of analogies used by science teachers. Jour. Res. Science Teaching, 32 (3), pp. 259- 270.

DANIELS, S. y BRAUND, M. (1992). Uncovering hierarchies in science assessment tasks. Int. Jour. Science Education, $14(4)$, pp. 463-474.

DEL CARMEN, L. (1995). Enfoques investigativos en la enseñanza y secuenciación de contenidos. Investigación en la Escuela, 25, pp. 17-25.

DÍAZ, J. y JIMÉNEZ, M.P.(1996). ¿Vesqué dibujas?. Observando células con el microscopio. Enseñanza de las Ciencias, 14(2), pp. 183-194.

DORAN, R., BOORMAN, J., CHAN, F. y HEJAILY, N. (1993). Alternative assessment of High School Iaboratory skills. Jour. Res. Science Teaching, 30(9), pp. 1121-1131.

DORAN, R., FRASER, B. y GIDDINGS, G. (1995). Science laboratory skills among grade 9 students in Western Australia. Int. Jour. Science Education, 17(1), pp. 27-44.

DUGGAN, S. y GOTT, R. (1995). The place of investigations in practical work in the UK National Curriculum for Science. Int. Jour. Science Education, 17(2), pp. 137-147.

DUGGAN, S., JOHNSON, P. y GOTT, R. (1996). A critical point in investigative work: defining variables. Jour. Res. Science Teaching, 33(5), pp. 461-474.

FRASER, B., GIDDINGS, G. y McROBBIE, C. (1995). Evolution and validation of a personal form of an instrument for assessing science laboratory classroom environments. Jour. Res. Science Teaching, 32 (4), pp. 399. 422.

FURIÓ, C., ITURBE, J. y REYES, J. (1994). Contribución de la resolución de problemas como investigación al paradigma constructivista de aprendizaje de las ciencias. Investigación en la Escuela, 24, pp. 89-99.

GALBRAITH, P., CARSS, M., GRICE, R., ENDEAN, L. y WARRY, $M$. (1997). Towards scientific literacy for the third miltennium: a view from Australia. Int. Jour. Science Education, 19(4), pp. 447-467.

GANGOLI, S. y GURUMURTHY, C. (1995). A study of the effectiveness of a guided open-ended approach to Physics experiments. Int. Jour. Science Education, 17(2), pp. $233-241$. 
GAYFORD, C. (1993). Discussion-based group work related to environmental issues in science classes with 15 -year-old pupils in England. Int. Jour. Science Education, 15(5), pp. 521-529.

GERMANN, P. y ARAM, R. (1996). Student performances on the Science processes of recording data, analyzing data, drawing conclusions, and providing evidence. Jour. Res. Science Teaching, 33(7), pp. 773-798.

GERMANN, P., ARAM, R. y BURKE, G. (1996a). Identifying patterns and relationships among the responses of SeventhGrade students to the Science process skill of designing experiments. Jour. Res. Science Teaching, 33(1), pp. 79-99.

GERMANN, P., HASKINS, S. y AULS, S. (1996b). Analysis of nine High School Biology laboratory manuals: promoting scientific inquiry, Jour. Res. Science Teaching, 33 (5), pp. $475+499$.

GIL, D. (1993). Contribución de la historia y de la filosofía de las ciencias al desarrollo de un modelo de enseñanza-aprendizaje como investigación. Enseñanza de las Ciencias, 11(2), pp. 197-212.

GIL, D. y VALDÉS, P. (1995). Un ejemplo de prăctica de laboratorio como actividad investigadora: segundo principio de la dinámica. Alambique, 6, pp. 93*102.

GILBERT, J. (1992). The interface between science education and technology education. Int. Jour. Science Education, 14(5), pp. 563-578.

GOTT, R. y DUGGAN, S. (1996). Practical work: its role in the understanding of evidence in science. Int. Jour. Science Education, 18(7), pp. 791-806

GRAYSON, D. (1997). A holistic approach to preparing disadvantaged students to succeed in tertiary science studies. Part H. Ottcomes of the Science Foundation Programe. Int. Jour. Science Education, 19(1), pp, 107-123.

HAFNER, R. y STEWART, J. (1995), Revising explanatory models to accommodate anomalous genetic phenomena: problem solving in the "context of discovery". Science Education, 79(2), pp. 111-146.

HANEY, J., CZERNIAK, C. y LUMPE, A. (1996). Teacher beliefs and intentions regarding the implementtion of Science Education reform strands. Jour. Res. Science Teaching, 33(9), pp. $971-993$

HARRISON, A. y TREAGUST, D. (1993). Teaching with analogies: a case study in grade 10 optics. Jour. Res. Science Teaching, 30(10), pp. 1291-1307.

HARRISON, A. y TREAGUST, D. (1996). Secondary students' mental models of atoms and molecules: implications for teaching Chemistry. Science Education, 80(5), pp. 509-534.

HODSON, D. (1994). Hacia un enfoque crítico del trabajo de laboratorio. Enseñanza de las Ciencias, 12(3), pp. 299-313.

HOLIDAY, W, YORE, L. y ALVERMAN, D. (1994). The reading-science learning-writing connection: breakthroughs, barriers, and promises. Jour. Res. Science Teaching, 31(8), pp. 877-893

HURST, R. y MILKENT, M. (1996). Faciliting successful prediction problem solving in Biology through application of skill theory. Jour. Res. Science Teaching, 33(S), pp. 541552 .

IZQUIERDO, M. (1994). La V de Gowin como instrumento para aprender a aprender ( $\mathrm{y}$ a pensar). Alambique, 1, pp. $114-124$.
JACKSON, D.,EDWARDS, B. y BERGER, C. (1993). Teaching the design and interpretation of graphs through computeraided graphical data analysis. Jour. Res. Science Teaching. 30(5), pp. 483-501.

KEYS, C. (1994). The development of scientific reasoning skills in conjunction with collaborative writing assignments: ant interpretative study of six 9 th-Grade students. Jour. Res. Science Teaching, 31(9), pp. 1003-1022.

KEYS, C. (1995). An interpretative study of students' use of scientific reasoning during a collaborative report writing intervention in Ninth Grade General Science. Science Education, 79(4), pp. 415-435.

KIRSCHNER, P., MEESTER, M., MIDDELBEEK, E. y HERMANS, H. (1993). Agreement between student expectations, experiences and actual objectives of practicals in the natural sciences at the Open university of The Netherlands. Int. Jour. Science Education, 15(2), pp. $175-197$

KOCH, A. y ECKSTEIN, S. (1995). Skills needed for reading comprehension of Physics texts and their relation to problemsolving ability. Jour. Res. Science Teaching, 32(6), pp. 613-628.

KUEH, $X$. (1992). Meaningful understanding of direct proportionality and consistency across different tasks among preservice science teachers. Int. Jour. Science Education, 14(2), pp. $237 \cdot 247$

LANGLOIS, F., GREA, J. y VIARD, J. (1995). Influencia de la formulación del enunciado y del control didáctico sobre la actividad intelectuai de los alumnos en resolución de problemas. Enseñanza de las Ciencias, 13(2), pp. $179-191$.

LAVOIE, D. (1993). The development, theory and application of a cognitive-network model of prediction problem solving in Biology. Jour. Res. Science Teaching, 30(7), pp. 767-785.

LAWSON, A. (1994). Uso de los ciclos de aprendizaje para la enseñanza de destrezas de razonamiento científico y de sistemas conceptuales. Enseñanza de las Ciencias, 12(2) pp. $165-187$

LEE, K. y FENSHAM, P. (1996). A general strategy for solving High School electrochemistry problems. Int. Jour. Science Education, 18(5), pp. 543-555.

LEE, K. GOH, N., CHIA, L. y CHIN, C. (1996). Cognitive variables in problem solving in Chemistry: a revisited study. Science Education, 80(6), pp. 691-710

LEE, O. y FRADD, S. (1996). Literacy skills in science learning among linguistically diverse students. Science Education, 80(6), pp. 651-671.

LEE, O., FRADD, S y SUTMAN, F. (1995). Science knowledge and cognitive strategy use among culturally and linguistically diverse students. Jour. Res. Science Teaching, 32(7), pp. 797- 816

LEONARD, W. (1992). A comparison of student performances following instruction by interactive videodisc versus conventional laboratory. Jour. Res. Science Education, 29(1), pp. 93-102.

LINDER, C. (1993). University Physics students' conceptualizations of factors affecting the speed of sound propagation. Int. Jour. Science Education, 15(6), pp. 655-662.

LOCK, R. (1992). Gender and practical skili performance in Science. Jour. Res. Science Teaching, 29(3), pp. 227- 241. 
LUBBEN, F, y MILLAR, R. (1996). Children's ideas about the reliability of experimental data. Int. Jour. Science Education, 18(8), pp. 955-968.

LUCAS, A. (1990). Varietes of science education research: their applications in the classroom. Enseñanza de las Ciencias, $8(3)$, pp. 205-214.

LUCAS, A. (1995). ¿Por qué utilizar el microscopio? Alambique, 6 , pp. 89-92.

MARTÍNEZ, M. y VARELA, P. (1996). De la resolución de problemas al cambio conceptual Investigación en la Escuela, 28, pp. 59-68.

MATTHEIS, F. et al. (1992). A study of the logical thinking skills and integrated process skills of Junior High School students in North Carolina and Japan. Science Education, $76(2)$, pp. $211-222$.

McNAY, M. y MELVILLE, K. (1993). Children's skill in making predictions and their understanding of what predicting means: a developmental study. Jour. Res. Science Teaching, 30(6), pp. 561-577.

MEC (1989). Secundaria Obligatoria. Ciencias de la Naturaleza. Madrid: MEC.

MESTRE, J., DUFRESNE, R., GERACE, W. y HARDIMAN, P. (1993). Promoting skilled problem-solving behavior among beginning Physics students. Jour. Res. Science Teaching, 30(3), pp. 303-317.

MONEREO, C. (1995). De los procedimientos a las estategias: implicaciones para el Proyecto Curricular Investigación y Renovación Escolar (IRES). Investigación en la Escuela, 27, pp. 21-38.

MUÑOZ, R. (1995). Escribir para aprender: ensayo de una alternativa en la enseñanza universitaria de las ciencias. Enseñanza de las Ciencias, 13(3), pp. 273-278.

NIAZ, M. (1996). Reasoning strategies of students in solving chemistry problems as a function of developmental leveI, functional $M$-capacity and disembedding ability. Int. Jour. Science Education, 18(5), pp. 525-541.

NIAZ, M. y ROBINSON, W. (1992). Manipulation of logical structure of Chemistry problems and its effect on student performance. Jour. Res. Science Teaching, 29(3), pp. 211226.

NIGRO, R. (1995). Un modelo de pruteba escrita que revela capacidades relacionadas con el proceso de aprendizaje. Enseñanza de las Ciencias, 13(3), pp. 347-361

NJOO, M. y JONG, T. (1993). Exploratory learning with a computer simulation for control theory: leaming processes and instructional support. Jour. Res. Science Teaching, 30(8), pp. 82i-844.

NOTT, M. y WELLINGTON, J. (1996). When the black box springs open: practical work in schools and nature of Science. Int. Jour. Science Education, 18(7), pp. 807-818.

NOVAK, J. (1991). Ayudar a los alumnos a aprender cómo aprender. La opinion de un profesor-investigador. Enseñanza de las Ciencias, 9(3), pp. 215-228.

PARK, J. y PAK, S. (1997). Students' responses to experimental evidence based on perceptions of causality and availability of evidence. Jour. Res. Science Teaching, 34(1), pp. 57-67.

PENNER, D., GILES, N., LEHRER, R. y SCHAUBLE, L (1997). Building functional models: designing an Elbow. Jour. Res. Science Teaching, 34(2), pp. 125-143.
PERALES, J. (1993). La resolución đe problemas: una revisión estructurada. Enseñanza de las Ciencias, 11(2), pp. $170 \cdot 178$.

PIZZINI, E. Y SHEPARDSON, D. (1992). A comparison of the classroom dynamics of a problem-solving and traditional laboratory model of instruction using path analysis. Jour. Res. Science Teaching, 29(3), pp. 243-258.

PREECE, J. y JANVIER, C. (1993). Interpreting trends in multiple-curve graphs of ecological situations: the role of context. Int. Jour. Science Education, 15(2), pp. 199-212.

PRO, A. (1995). Reflexiones para la selección de contenidos procedimentales en ciencias. Alambique, 6, pp. 77-87

PRO, A. (1997). ¿Cómo pueden secuenciarse los contenidos procedimentales? Alambique, 14 (en prensa).

RITCHIE, S. y RIGANO, D. (1996). Laboratory apprenticeship through a student research project. Jour. Res. Science Teaching, 33(7), pp. 799-815.

ROBERTS, D. (1995). Junior high school science transformed: analysing a science curriculum policy change. Int. Jour. Science Education, 17(4), pp, 493-504.

ROTH, W. (1994). Experimenting in a constructivist High School Physics laboratory. Jour. Res. Science Teaching, 31(2), pp. 197-223.

ROTH, W.y BOWEN, G. (1994). Mathematization of experience in a Grade 8 open-inquiry environment: an introduction to thr representational practices of Science. Jour. Res. Science Teaching, 31(3), pp. 293-318.

ROTH, W. y McGINN, M. (1997). Graphing: cognitive abjlity or practice? Science Education, 81(1), pp. 91-106.

ROTH, W. y ROYCHOUDHURY, A. (1993). The development of science process skills in authentic contexts. Jour. Res. Science Teaching, 30(2), pp. 127-152.

SANMARTÍ, N. (1997). Enseñar a elaborar textos científicos en las clases de ciencias. Alambique, 12, pp. 51-61.

SEROUSSI, D. (1995). Heuristic hypotheses in problem solving: an example of conceptual issues about scientific procedures. Science Education, 79(6), pp. 595-609.

SEVILLA, C. (1994). Los procedimientos en el aprendizaje de la física. Enseñanza de las Ciencias, 12(3), pp. 400-405.

SHAYER, M. y ADEY, P. (1984). La ciencia de enseñar ciencias. Madrid: Narcea.

SHEPARDSON, D., MOJE, E. y KENNARD, A. (1994). The impact of a science demonstration on children's understanding of air pressure. Jour. Res. Science Teaching, 31(3), pp. 243-258.

SHEPARDSON, D. y PIZZINI, E. (1992). Gender bias in female elementary teachers' perceptions of the scientific ability of students. Science Education, 76(2), pp. 147-153.

SIGÜENZA, A. y SÁEZ, M.J. (1990). Análisis de la resolución de problemas como estrategia de enseñanza de la biología. Enseñanza de las Ciencias, 8(3), pp. 223-230.

SIMMONS, P. y LUNETTA, V. (1993). Problem-solving behaviors during a Genetics computer simulation: beyond the expert/novice dichotomy. Jour. Res. Science Teaching, $30(2)$, pp. 153-173.

SONG, J, y BLACK, P. (1992). The effects of concepts, requirements and task contexts on pupils' performance in 
control of variables. Int. Jour. Science Education, 14(1), pp. 83-93.

STAVY, R, y TIROSH, D. (1996). Intuitive rules in science and mathematics: the case of 'more of A-more of B'. Int. Jour. Science Education, 18(6), pp. 653-667.

STRANG, J. y SHAYER, M. (1993). Enhancing High School students' achievement in Chemistry through a thinking skills approach. Int. Jour. Science Education, 15(3), pp. 319-337.

TAMIR, P.y GARCIA M.P. (1992). Characteristics of laboratory exercises included in science textbooks in Catalonia (Spain). lnt. Jour. Science Education, 14(4), pp. 381-392.

TREAGUST, D., HARRISON, A., VENVILLE, G. y DAGHER, $Z$. (1996). Using an analogical teaching approach to engender conceptual change. Int. Jour. Science Education, 18(3), pp. 213-219.

VARELAS, M. (1996). Between theory and data in a SeventhGrade Science class. Jour. Res. Science Teaching, 33(3), pp. 229-263.
WATSON, J. (1994), Students' engagement in practical problem solving: a case study. Int. Jour. Science Education, 16(1), pp. 27-43.

WENHAM, M. (1993). The nature and role of hypotheses in school science investigations. Int. Jour. Science Education, 15(3), pp. 23I-240.

WESTBROOK, S. y ROGERS, L. (1994). Examining the development of scientific reasoning in Ninth-Grade Physical Science students. Jour. Res. Science Teaching, 31(1), pp. $65-76$.

WHITE, R. (1996). The link between the laboratory and leaming. Int. Jour. Science Education, 18(7), pp. 761-774.

ZAJCHOWSKI, R. y MARTIN, J. (1993). Differences in the problem solving of stronger and weaker novices in Physics: knowledge, strategies, or knowledge, structure? Jour. Res. Science Teaching, 30(5), pp. 459-470.

ZOHAR, A. (1995). Reasoning about interactions between variables. Jour. Res. Science Teaching, 32(10), pp. 10391063. 